\title{
Inhibition of HDAC1 alleviates monocrotaline-induced pulmonary arterial remodeling through up-regulation of miR-34a
}

\author{
Fangwei Li®, Dan Wang, Hong Wang, Lijun Chen, Xilu Sun and Yixin Wan*
}

\begin{abstract}
Background: It has been found that up-regulation of histone deacetylases 1 (HDAC1) is involved in the development of pulmonary arterial hypertension (PAH). However, it is still unclear whether inhibition of HDAC1 suppresses the development of PAH via restoring miR-34a level in monocrotaline (MCT)-induced PAH rats.

Methods: PAH rat models were induced by intraperitoneal injection of MCT. HDAC1 was suppressed by intraperitoneal injection of the class I HDAC inhibitor MS-275, and miR-34a was over-expressed via tail vein injection of miR-34a agomiR.

Results: HDAC1 protein was significantly increased in MCT-induced PAH rats; this was accompanied with downregulation of miR-34a and subsequent up-regulation of matrix metalloproteinase 9 (MMP-9)/tissue inhibitor of metalloproteinase 1 (TIMP-1) and MMP-2/TIMP-2. Administration of PAH rats with MS-275 or miR-34a agomiR dramatically abolished MCT-induced reduction of miR-34a and subsequent up-regulation of MMP-9/TIMP-1 and MMP-2/TIMP-2, finally reduced extracellular matrix (ECM) accumulation, pulmonary arterial remodeling, right ventricular systolic pressure (RVSP) and right ventricle hypertrophy index (RVHI) in PAH rats.

Conclusions: HDAC1 contributes to the development of MCT-induced rat PAH by suppressing miR-34a level and subsequently up-regulating the ratio of MMP-9/TIMP-1 and MMP-2/TIMP-2. Inhibition of HDAC1 alleviates pulmonary arterial remodeling and PAH through up-regulation of miR-34a level and subsequent reduction of MMP-9/TIMP-1 and MMP-2/TIMP-2, suggesting that inhibition of HDAC1 might have potential value in the management of PAH.
\end{abstract}

Keywords: HDAC1, Extracellular matrix, miR-34a, Pulmonary arterial hypertension

\section{Background}

Pulmonary arterial hypertension (PAH) is defined as a hemodynamic state of the resting mean pulmonary arterial pressure equal to or more than $25 \mathrm{mmHg}$, leading to right heart failure and ultimately death [1]. Pulmonary vascular remodeling is the prominent hallmark of $\mathrm{PAH}$, in which the accumulation of extracellular matrix (ECM) with increased collagen deposition is an indispensable

\section{*Correspondence: wanyixinzr@163.com}

Department of Respiratory Medicine, Lanzhou University Second Hospital, No. 82, Cuiying Men, Lanzhou 730030, Gansu, People's Republic of China change across the vascular wall [2, 3]. Excessive accumulation of ECM is convinced to occur due to the deregulation of multiple signaling pathways in pulmonary arterial smooth muscle cells (PASMCs) [4]. Therefore, it is important to inhibit the cascades responsible for collagen synthesis or activate the signaling pathways associated with collagen degradation in the prevention and treatment of PAH.

Histone acetylation is dynamically regulated by histone acetyltransferases (HATs) and histone deacetylases (HDACs), playing an important role in controlling gene expression [5]. HDACs remove acetyl groups from the lysine residues of histones and cause dense crispation of 
chromatin, leading to the suppression of gene transcription; while HATs result in the opposite process [6]. There are 18 HDACs which are grouped into four classes and HDAC1 belongs to the class I HDACs [6]. Class I HDACs (HDAC1, 2, 3, and 8) play an important role in controlling cell cycle regulation, ECM accumulation and tissue development by deacetylating substrates and then regulating gene expression [7-9]. Studies have shown that the overall activity of HDACs is determined by the expression level of HDAC1 to some extent [10].

Recent studies have found that HDAC1 is up-regulated in lung tissues of patients with PAH and model rats [11, 12]; selective class I HDAC inhibitors reverse monocrotaline (MCT) and hypoxia-induced pulmonary arterial remodeling and PAH by reducing HDAC1 expression [13]. Class I HDACs participate in ECM remodeling of various tissues through multiple molecular mechanisms $[9,14]$. In addition, studies have suggested that HDAC1 inhibits miR-34a expression directly or indirectly [15, 16] and down-regulation of miR-34a plays an important role in the pathogenesis of PAH $[17,18]$. However, whether inhibition of HDAC1 alleviates pulmonary arterial remodeling and PAH via suppressing excessive ECM accumulation is not defined. In addition, whether inhibition of HDAC1 restores miR-34a expression and thereby reduces excessive ECM accumulation needs further exploration. In the current study, rat models of MCTinduced PAH were used to evaluate the effects of HDAC1 on ECM remodeling of the pulmonary artery and to further explore its potential molecular mechanisms.

\section{Methods}

\section{Animals}

Forty male Sprague-Dawley (SD) rats weighing 150$200 \mathrm{~g}$ used in this study were purchased from Lanzhou Veterinary Research Institute, Chinese Academy of Agricultural Sciences. Rats were kept under standard conditions $\left(18-22{ }^{\circ} \mathrm{C}\right.$ and $40-60 \%$ humidity) in a specific pathogen free animal laboratory, and they had free access to food and water according to the U.S. National Research Council's Guide for the Care and Use of Laboratory Animals. Forty rats were divided into four groups randomly $(\mathrm{n}=10$ in each group): control group, $\mathrm{MCT}$ group, MCT + MS-275 group, and MCT + miR-34a agomiR group.

\section{Chemical reagents}

MCT (Sigma-Aldrich, St. Louis, MO, USA) was dissolved in $1 \mathrm{~mol} / \mathrm{L} \mathrm{HCL}$ and then titrated with $1 \mathrm{~mol} / \mathrm{L} \mathrm{NaOH}$ to $\mathrm{pH} 7.4$ with a final concentration of $30 \mathrm{mg} / \mathrm{mL}$. Class I HDAC inhibitor MS-275 (Selleck Chemicals, USA) was dissolved in dimethyl sulfoxide (DMSO) and then diluted with normal saline to a final concentration of $6 \mathrm{mg} / \mathrm{mL}$.
miR-34a agomiR (GenePharm, Shanghai, China) was diluted with diethyl pyrocarbonate (DEPC) to a final concentration of $25 \mu \mathrm{M}$.

\section{Generation of rat PAH and reagent intervention models}

The rat PAH model was induced by intraperitoneally injection of MCT $(60 \mathrm{mg} / \mathrm{kg})$ on day 1. MS-275 $(3 \mathrm{mg} / \mathrm{kg})$ was administrated to rats by intraperitoneally injection every other day for 28 days after MCT injection. miR34 a agomiR $(25 \mu \mathrm{M})$ was given to rats via tail vein injection on days $1,7,14,21$, and 28 respectively after MCT injection.

\section{Hemodynamic and right ventricular hypertrophy evaluation}

After 28 days of MCT injection, all survived rats were anesthetized with $10 \%$ chloral hydrate $(3-5 \mathrm{~mL} / \mathrm{kg})$ via intraperitoneal injection. Then the rats were put in supine position and a polyvinyl catheter filled with heparin saline solution were inserted into the right ventricle via the isolated right external jugular vein. Finally, the right ventricle systolic pressure (RVSP) was measured using a Grass polygraph (Power Lab, Australia). After completion of hemodynamic measurements, the hearts and lungs were harvested. Then the right ventricle (RV) was dissected from the left ventricle (LV) plus interventricular septum (S). Each part was weighed separately to calculate the right ventricular hypertrophy index (RVHI), which is the ratio of the weight of RV to the LV plus $S$ $[\mathrm{RV} /(\mathrm{LV}+\mathrm{S})]$.

\section{Histological analysis}

The harvested right upper pulmonary lobes were fixed using $4 \%$ paraformaldehyde for $24 \mathrm{~h}$ at room temperature and then embedded in paraffin wax. The paraffin embedded tissue blocks were sectioned at $5 \mu \mathrm{m}$ thickness and then stained with hematoxylin and eosin (HE) staining as well as Masson's trichrome staining for morphological analysis. To evaluate the degree of pulmonary arterial remodeling, the distance between the inner and outer elastic fiber layers (medial wall thickness, MT) and the average diameter of the outer elastic fiber layer (external diameter, ED) were measured in HE staining under a light microscope $(\times 400$ magnification). Then the percentage of medial wall thickness (\%MT) of vessels was calculated as follows $[19,20]: \% \mathrm{MT}=(2 \times \mathrm{MT} / \mathrm{ED}) \times 100 \%$.

\section{Immunofluorescence staining}

The paraffin sections were prepared using the same method as in HE staining, on which the immunofluorescence staining of rat lung tissues was performed. The sections were blocked with $3 \%$ bovine serum albumin (BSA) to avoid nonspecific immunoreactions and then 
incubated overnight at $4{ }^{\circ} \mathrm{C}$ with primary antibody of HDAC1 (CST, Beverly, MA, USA; 1:1000). After being washed three times, the sections were incubated at $37^{\circ} \mathrm{C}$ for $2 \mathrm{~h}$ with goat anti-rabbit secondary antibody (SigmaAldrich; 1:5000). Subsequently, the sections were stained by 4',6-diamidino-2-phenylindole (DAPI) for $5 \mathrm{~min}$ at room temperature. In addition, negative control immunofluorescence was conducted to show the auto-fluorescence using equal amount of phosphate buffered solution (PBS) instead of the primary antibody and secondary antibody. Finally, fluorescence images were randomly scanned using a confocal laser scanning microscope at $\times 400$ magnification.

\section{Cell culture}

Primary cultured PASMCs were obtained from the pulmonary arteries of 4- to 5-week-old male SD rats. All animal experiments were approved by the Laboratory Animal Care Committee of Lanzhou University Second Hospital and performed in accordance with the Guidelines for Animal Experimentation of Lanzhou University Second Hospital. The pulmonary arteries were rapidly removed from euthanized rats and the layers of adventitia as well as endothelium were removed gently. Then the remaining smooth muscle layer was minced $\left(\sim 1 \mathrm{~mm}^{2}\right)$ and placed into a culture flask with Dulbecco's Modified Eagle Medium (DMEM, Gibco, Grand Isle, NY, USA) containing 10\% fetal bovine serum (FBS, Sijiqing, HangZhou, China) as well as $1 \%$ antibiotics $(100 \mathrm{U} / \mathrm{mL}$ penicillin and $100 \mu \mathrm{g} / \mathrm{mL}$ streptomycin). The tissue blocks were cultured at $37{ }^{\circ} \mathrm{C}$ in an atmosphere of $95 \%$ air and $5 \% \mathrm{CO}_{2}$. PASMCs were sub-cultured using $0.25 \%$ trypsin (Invitrogen, Carlsbad, CA, USA) when reaching $80 \%$ confluence, and cells between passages 4 and 6 were used for cell studies.

\section{siRNA transfection}

Cell transfection was performed for knockdown the expression of HDAC1 using Lipofectamine ${ }^{\mathrm{TM}} 2000$ transfection reagent (Invitrogen) according to the manufacturer's protocols. PASMCs were cultured in a 6-well plate until reaching $30-40 \%$ confluence. The nucleotides of HDAC1 siRNA were used at a final concentration of $100 \mathrm{nM}$ in DMEM (without serum or antibiotics) for $6 \mathrm{~h}$ and complete DMEM with 10\% FBS (without antibiotics) for $48 \mathrm{~h}$ in a $37{ }^{\circ} \mathrm{C}, 5 \% \mathrm{CO}_{2}$ humidified incubator. Transfection efficiency of siRNA was determined using immunoblotting.

\section{ELISA}

The collagen I level in harvested peripheral lung tissues was measured using a rat collagen I enzyme-linked immunosorbent assay (ELISA) kit (Xitang, Shanghai,
China) according to the manufacturer's instructions. Briefly, the lung tissues were homogenized in $0.9 \% \mathrm{NaCl}$ on ice and centrifuged at $6000 \mathrm{rpm}$ for $20 \mathrm{~min}$ at $4{ }^{\circ} \mathrm{C}$. Then the supernatant was collected and the concentration of sample was calculated automatically by the instrument software according to the measured optical density values. Duplicate samples were assayed, and all results were reported as the mean values.

\section{Immunoblotting}

The left lung tissues were homogenized in RIPA lysis buffer (HEART, Xi'an, China) containing protease inhibitors, phosphatase inhibitors as well as phenylmethylsulfonyl fluoride (PMSF), and centrifuged at $12,000 \mathrm{rpm}$ at $4{ }^{\circ} \mathrm{C}$ for $20 \mathrm{~min}$. Then the supernatant was collected as total protein. The amount of protein in each extract was measured using BCA protein assay kit (Pierce Biotechnology, Rockford, IL, USA). Subsequently, equal amounts of protein extracts were separated on SDS-PAGE gel $(10 \%)$ and transferred to polyvinylidene fluoride (PVDF, Bio-Rad, Richmond, CA, USA) membrane using wet transfer. After washing, the blots were incubated at $4{ }^{\circ} \mathrm{C}$ overnight with the primary antibodies against HDAC1 (CST; 1:1000), HDAC2 (CST; 1:1000), HDAC3 (CST; 1:1000), tissue inhibitor of metalloproteinase 1 (TIMP1, Abcam, Cambridge, UK; 1:1000), TIMP-2 (Abcam; 1:1000) and $\beta$-actin (Santa Cruz, Dallas, TEX, USA; 1:500). Then the horseradish peroxidase-conjugated goat anti-rabbit IgG (Sigma-Aldrich; 1:5000) was used as the secondary antibodies. Finally, immunoreactive bands were visualized by Super Signal West Pico Chemiluminescent Substrate (Pierce Biotechnology) and quantified by Quality One software (Bio-Rad).

\section{qRT-PCR}

Total RNA was extracted from lung tissues using TRIzol reagent (Invitrogen) according to the manufacturer's instructions. U6 small nuclear RNA was chosen for endogenous control of miR-34a. Complementary DNA (cDNA) was synthesized using the RevertAid First Strand cDNA Synthesis Kit (Thermo, Waltham, MA, USA) and quantitative real-time polymerase chain reaction (qRTPCR) was carried out using the Maxima SYBR Green/ ROX qPCR Master Mix (Thermo). miR-34a was quantified by measuring cycle threshold $(\mathrm{Ct})$ values and normalized using $2^{-\Delta \Delta C t}$ method relative to U6. Primers (Sangon Biotech, Shanghai, China) for miR-34a and U6 were as follows:

miR-34a RT, 5'-GTCGTATCCAGTGCGTGTCGT GGAGTCGGCAATTGCACT

GGATACGACTAGGGC-3';

miR-34a forward, 5'-CGCGAATCAGCAAGTATA ACTGCC-3'; 
miR-34a reverse, 5'-CGTATCCAGTGCGTGTCG TGG-3';

\section{U6 RT, CGCACTGGATACGACGGCATTCT;} U6 forward, CTCGCTTCGGCA GCACA; U6 reverse, AACGCTTCACGAATTTGCGT.

\section{HDAC activity}

Nuclear extracts were obtained from rat lung tissues (20 mg) using the Nuclear Extract kit (Active Motif, Carlsbad, CA, USA) according to the manufacturer's instructions. HDAC activity was measured in the nuclear extracts with the Fluorometric HDAC Activity Assay Kit (Abnova, walnut, Los Angeles, USA) in accordance with the manufacturer's instructions. Briefly, $15 \mu \mathrm{g}$ nuclear extracts was diluted in assay buffer and trichostatin A, a classic HDAC inhibitor as the negative control. Then the HDAC reaction was initiated by addition of fluorometric substrate and the mixture was incubated at $37^{\circ} \mathrm{C}$ for $30 \mathrm{~min}$. Finally, the reaction was stopped by adding lysine developer for $30 \mathrm{~min}$ and the sample was measured using a fluorescence plate reader with excitation at $380 \mathrm{~nm}$ and emission at $460 \mathrm{~nm}$ (Bio-Rad). The HDAC activity was expressed as relative fluorescence units.

\section{Gelatin zymography}

The enzymatic activity of matrix metalloproteinase $2 / 9$ (MMP-2/9) was determined by gelatin zymography. Rat lung tissues were homogenized in Tris- $\mathrm{HCl}$ buffer containing $50 \mathrm{mM}$ Tris- $\mathrm{HCl} \mathrm{pH}$ 7.6, $150 \mathrm{mM} \mathrm{NaCl}, 5 \mathrm{mM} \mathrm{CaCl}$, and $0.1 \%$ Triton X-100. Equal amounts of protein were separated on gelatin zymography gel by electrophoresis. Next, the gel was incubated for $48 \mathrm{~h}$ in development buffer containing $50 \mathrm{mM}$ Tris- $\mathrm{HCl} \mathrm{pH} \mathrm{7.6,} 5 \mathrm{mM} \mathrm{CaCl}_{2}$, and $1 \mu \mathrm{M} \mathrm{ZnCl}$ at $37{ }^{\circ} \mathrm{C}$. Then the gel was stained with $0.5 \%$ Coomassie Blue R-250 for $3 \mathrm{~h}$ and destained in destaining buffer containing $30 \% \mathrm{MeOH}$ and $10 \%$ acetic acid. Finally, Gel Doc ${ }^{\mathrm{TM}}$ XR (Bio-Rad) was used to visualize and analyze the resulting bands.

\section{Statistics}

The statistical analysis was performed by SPSS 13.0 software (SPSS Inc., Chicago, IL, USA). Values were presented as mean \pm standard deviation. Group comparisons were performed using one-way analysis of variance (ANOVA) followed by Dunnett post hoc test (between the control group and treatment groups) or Student-Newman-Keuls post hoc test (among different groups). $P<0.05$ was considered to be statistically significant.

\section{Results}

Inhibition of HDAC1 ameliorates the increase of RVSP and RVHI in MCT-induced PAH rats

Protocol for generation of rat $\mathrm{PAH}$ and reagent intervention models was shown in Fig. 1. After 28 days of MCT injection, the RVSP in MCT-treated rats was significantly increased compared with the control group $(56.45 \pm 2.54 \mathrm{mmHg}$ versus $21.70 \pm 1.70 \mathrm{mmHg}, P<0.05)$, suggesting that PAH was successfully induced by MCT in rats. However, treatment with class I HDAC inhibitor MS-275 after MCT injection dramatically reduced RVSP to $28.69 \pm 1.42 \mathrm{mmHg}(P<0.05$ versus MCT group), as depicted in Fig. 2A and B. Similar changes were observed in RVHI. Figure $2 \mathrm{C}$ showed that the $\mathrm{RV} /(\mathrm{LV}+\mathrm{S})$ ratio was elevated from $0.23 \pm 0.02$ in the control to $0.57 \pm 0.05$ in MCT-induced PAH rats $(P<0.05)$. However, after administration of MS-275 in rats exposed to MCT, the $\mathrm{RV} /(\mathrm{LV}+\mathrm{S})$ ratio was declined to $0.35 \pm 0.04 \quad(P<0.05$ versus MCT group). These results suggest that inhibition of HDAC1 suppresses the development of PAH.

\section{HDAC1 is increased in MCT-induced PAH rats}

The protein level of HDAC1 in the lung tissues of MCTtreated rats was detected by immunofluorescence staining and immunoblotting to determine the potential role of HDAC1 in PAH. As indicated in Fig. 3A, the fluorescence-labeled HDAC1 in pulmonary vessels with diameters both greater than $50 \mu \mathrm{m}$ and less than $50 \mu \mathrm{m}$ of MCT-treated rats showed a significant increase compared with the control rats, while MS-275 treatment reduced fluorescence-labeled HDAC1 in pulmonary vessels with diameters both greater than $50 \mu \mathrm{m}$ and less than $50 \mu \mathrm{m}$ of MCT-treated rats. As expected in Fig. 3B, the protein level of HDAC1 was up-regulated to 2.30-fold in MCT-treated rats compared with the control rats $(P<0.05)$, while administration of MS-275

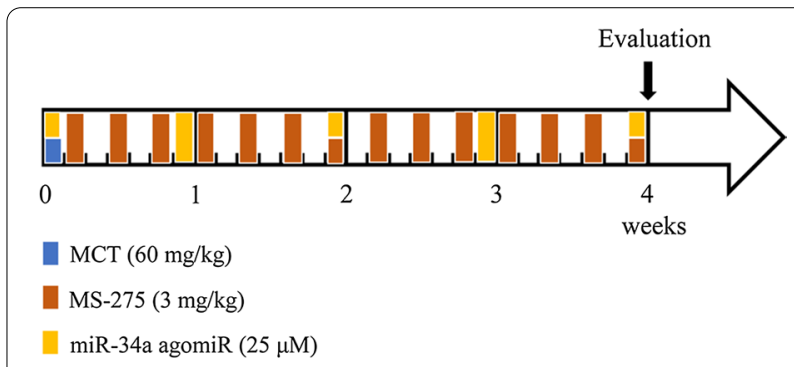

Fig. 1 Protocol for generation of rat pulmonary arterial hypertension $(\mathrm{PAH})$ and reagent intervention models. The rat PAH model was induced by intraperitoneally injection of monocrotaline (MCT) ( $60 \mathrm{mg} / \mathrm{kg}$ ) on day 1. MS-275 (3 mg/kg) was administrated to rats by intraperitoneally injection every other day for 28 days after MCT injection. miR-34a agomiR $(25 \mu \mathrm{M})$ was given to rats via tail vein injection on days 1, 7, 14, 21 and 28 respectively after MCT injection 


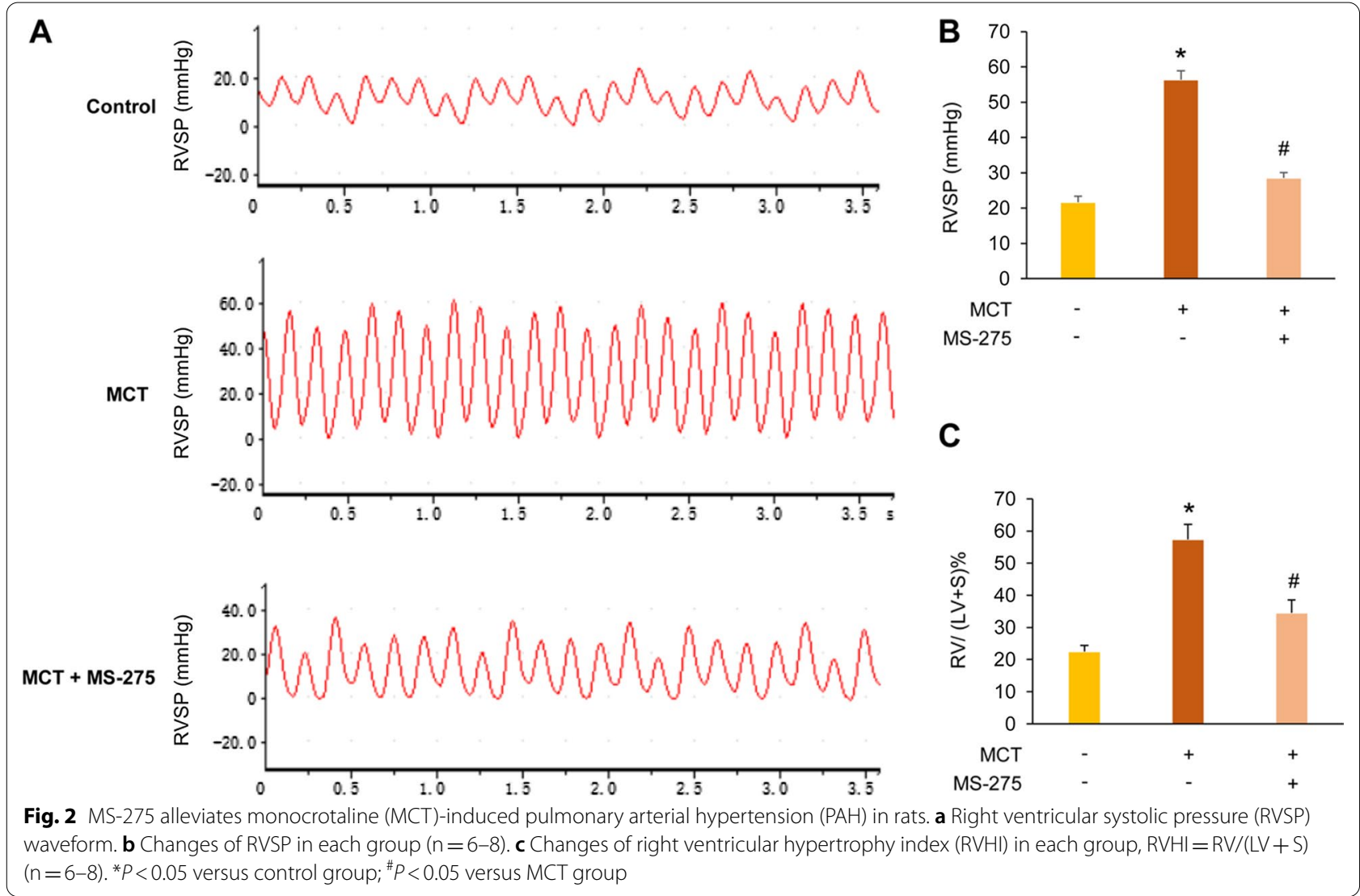

down-regulated HDAC1 level of MCT-treated rats to 1.15 -fold over control $(P<0.05$ versus $\mathrm{MCT}$ group). The protein levels of HDAC2 and HDAC3 remained unchanged in MCT-treated rats compared with the control rats $(P>0.05)$ and were not affected by administration of MS-275 in rats exposed to MCT $(P>0.05$ versus $\mathrm{MCT}$ group). These results suggest that HDAC1 is significantly increased and can be inhibited by MS-275 in MCT-induced PAH rats.

\section{Inhibition of HDAC1 restores miR-34a expression in MCT-induced PAH rats and PASMCs}

To determine whether HDAC1 down-regulates miR-34a through deacetylation in MCT-induced PAH rats, the expression of miR-34a and the activity of HDAC were examined. As shown in Fig. 4A, MCT obviously reduced miR-34a level to 0.62-fold compared with control group $(P<0.05)$, while treatment of MCT-induced $\mathrm{PAH}$ rats with class I HDAC inhibitor MS-275 restored the miR34 a expression to 0.89 -fold over control $(P<0.05$ versus MCT group). Furthermore, the MCT-treated rats showed increased HDAC activity compared with the control group $(9.52 \pm 0.35 \mathrm{RFU} / \mu \mathrm{g}$ versus $3.62 \pm 0.25 \mathrm{RFU} / \mu \mathrm{g}$, $P<0.05)$, while administration of $\mathrm{MCT}$-induced $\mathrm{PAH}$ rats with MS-275 significantly reduced HDAC activity to $6.8 \pm 0.15 \mathrm{RFU} / \mu \mathrm{g}(P<0.05$ versus $\mathrm{MCT}$ group$)$, as presented in Fig. 4B. These findings indicate that inhibition of HDAC1 restores miR-34a expression through reducing deacetylation in MCT-induced PAH rats.

In addition, HDAC1 siRNA was transfected into the rat primary cultured PASMCs to verify the specific role of HDAC1 in regulation of miR-34a. Figure 4C showed that HDAC1 siRNA reduced HDAC1 protein level to $39 \%$ of the control group $(P<0.05)$, whereas control siRNA did not affect HDAC1 protein level. Figure 4D presented that silencing of HDAC1 dramatically up-regulated miR-34a level, which increased to 1.96-fold compared with control group $(P<0.05)$, while the non-targeting siRNA had no effect on miR-34a expression. These results suggest that knockdown of HDAC1 is particularly responsible for miR-34a up-regulation in PASMCs.

Inhibition of HDAC1 reduces the ratio of MMP-9/TIMP-1 and MMP-2/TIMP-2 and the expression of collagen I through restoring miR-34a level in MCT-induced $\mathrm{PAH}$ rats To further investigate the mechanism of HDAC1 mediation of MCT-induced collagen deposition, the activity of MMP-2/9 and the expression of TIMP-1/2 were 


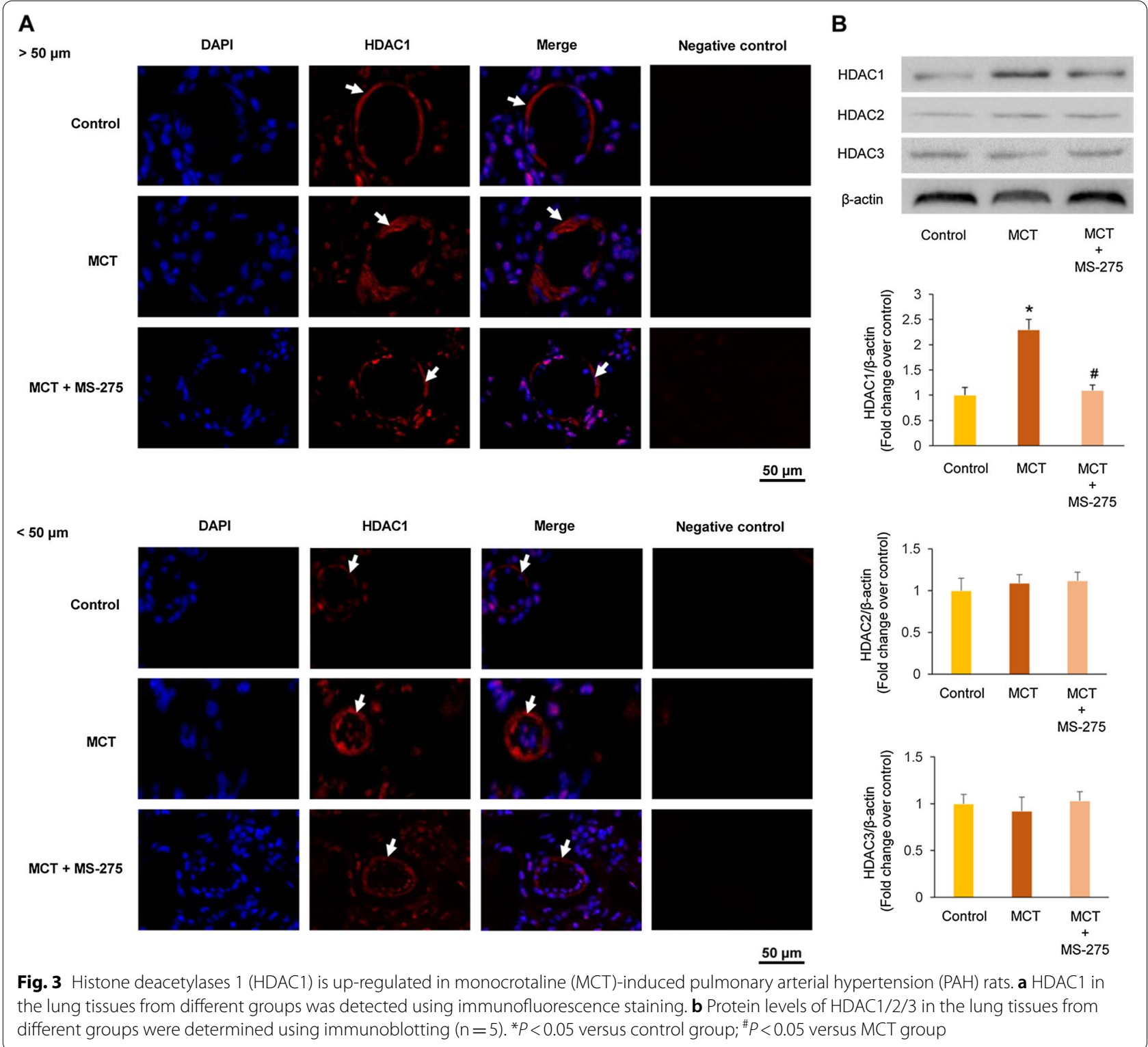

examined in the lung tissues of rats. As shown in Fig. 5A, miR-34a level was down-regulated in MCT-induced PAH model, which was 0.51 -fold over control $(P<0.05)$; while treatment of MCT-induced PAH rats with miR34a agomiR up-regulated miR-34a expression, which was 1.18 -fold over control ( $P<0.05$ versus MCT group). Figure $5 \mathrm{~B}$ indicated that $\mathrm{MCT}$ induced a 3.10 -fold increase over control in MMP-2 activity $(P<0.05)$ and a 2.51 fold increase over control in MMP-9 activity $(P<0.05)$. MS-275 treatment decreased MMP-2/9 activity in MCTtreated PAH rats to 1.20 -/1.10-fold over control $(P<0.05$ versus MCT-treated group), and administration of miR34a agomiR reduced MMP-2/9 activity in MCT-treated rats to $1.15-/ 1.04$-fold over control $(P<0.05$ versus $\mathrm{MCT}$ group). Figure $5 \mathrm{C}$ showed that the protein levels of TIMP-1 and TIMP-2 increased to 1.60-fold and 1.85-fold over control in the MCT-treated rats $(P<0.05)$, respectively; whereas treatment of MCT-induced $\mathrm{PAH}$ rats with MS-275 decreased TIMP-1/2 protein levels to 0.92-/0.90fold over control $(P<0.05$ versus MCT-treated group), and administration of miR-34a agomiR reduced TIMP$1 / 2$ protein levels in MCT-treated rats to 0.85-/0.92fold over control $(P<0.05$ versus MCT group). Finally, the ratio of MMP-9/TIMP-1 and MMP-2/TIMP-2 was analyzed. As presented in Fig. 5D, the ratio of MMP-9/ TIMP-1 and MMP-2/TIMP-2 was 1.57-/1.60-fold over 
A
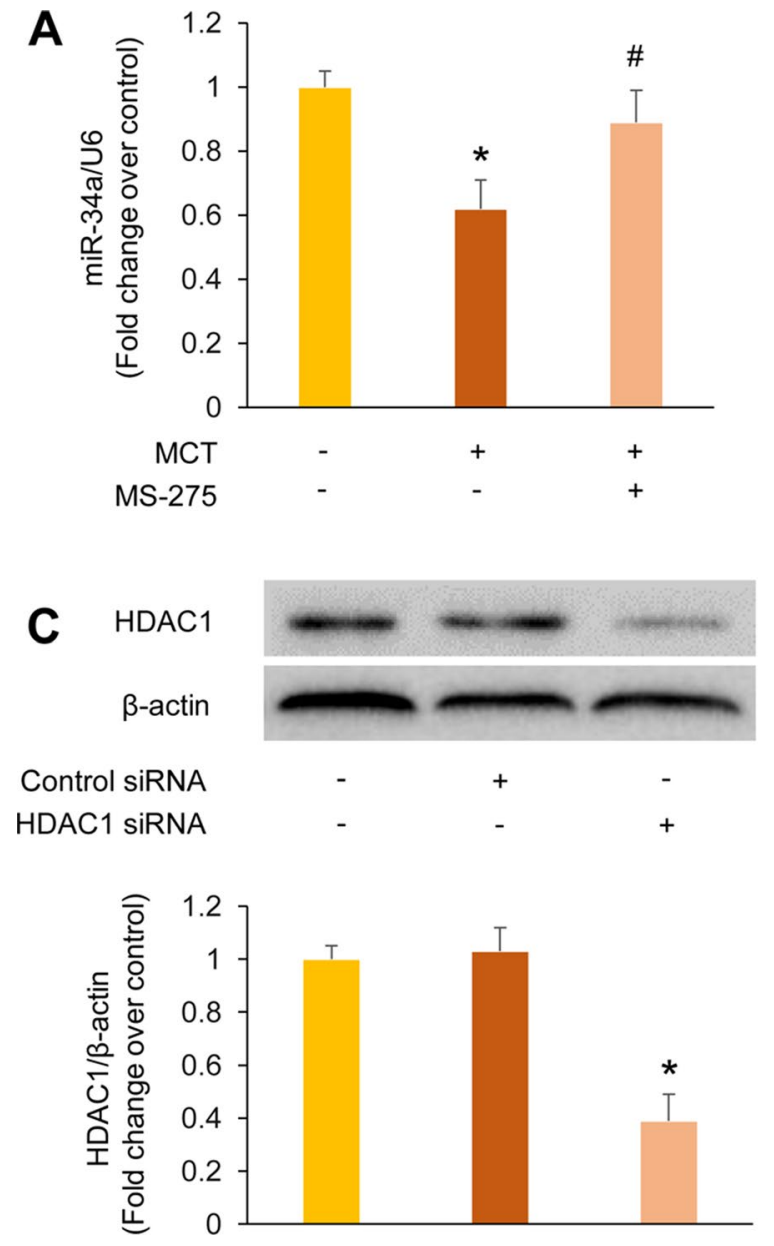

B
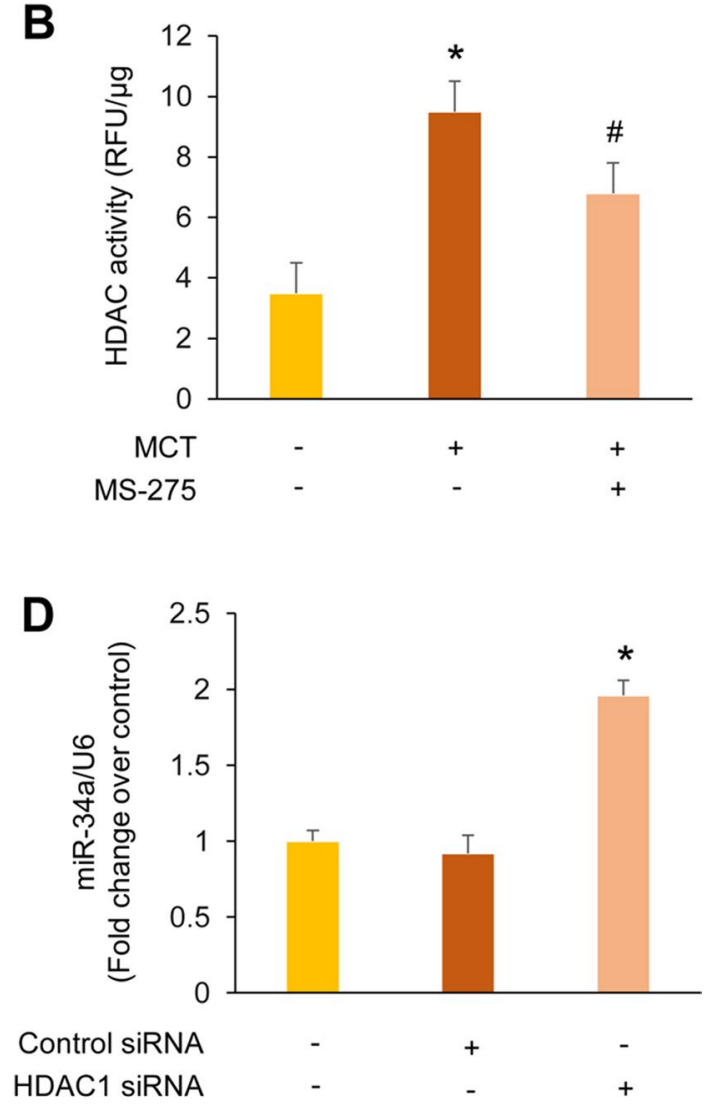

Fig. 4 Inhibition of histone deacetylases 1 (HDAC1) restores miR-34a level in monocrotaline (MCT)-induced pulmonary arterial hypertension (PAH) rats and pulmonary arterial smooth muscle cells (PASMCs). a miR-34a level in lung tissues from different groups was determined using quantitative real-time polymerase chain reaction (qRT-PCR) $(n=3)$. $\mathbf{b}$ HDAC activity in lung tissues from different groups was measured with a fluorometric HDAC activity assay kit $(n=3)$. c Protein level of HDAC1 in PASMCs transfected with HDAC1-specific siRNA or control siRNA was examined using immunoblotting $(n=3)$. d miR-34a level in PASMCs transfected with HDAC1-specific siRNA or control siRNA was tested using qRT-PCR $(n=3)$.

${ }^{*} P<0.05$ versus control group; ${ }^{\#} P<0.05$ versus MCT group

control in MCT-treated rats $(P<0.05)$; MS-275 treatment decreased the ratio of MMP-9/TIMP-1 and MMP-2/ TIMP-2 in MCT-treated PAH rats to 1.20-/1.30-fold over control $(P<0.05$ versus MCT-treated group), and administration of miR-34a agomiR reduced the ratio of MMP-9/TIMP-1 and MMP-2/TIMP-2 in MCT-treated rats to $1.22-/ 1.25$-fold over control $(P<0.05$ versus MCT group).

Then the expression level of collagen I in the lung tissues was examined by ELISA. As depicted in Fig. 5E, MCT group showed a 3.90-fold increase of collagen I compared with control group $(P<0.05)$. However, treatment of MCT-induced PAH rats with MS-275 and miR$34 \mathrm{a}$ agomiR markedly decreased the collagen I level to 2.66-fold and 1.86-fold compared with control group, respectively (both $P<0.05$ versus $\mathrm{MCT}$ group). These results suggest that inhibition of HDAC1 reduces the ratio of MMP-9/TIMP-1 and MMP-2/TIMP-2 and the expression of collagen I through restoring miR-34a level in MCT-induced PAH rats.

\section{Inhibition of HDAC1 suppresses pulmonary arterial} remodeling, excessive ECM accumulation and the increase of RVSP and RVHI through restoring miR-34a level in MCT-induced PAH rats

Pulmonary arterial remodeling plays an extremely critical role in the pathological progress of PAH. The HE staining results indicated that no matter pulmonary vessels with diameters greater than $50 \mu \mathrm{m}$ or less than $50 \mu \mathrm{m}$, both MS-275 and miR-34a agomiR markedly reversed 


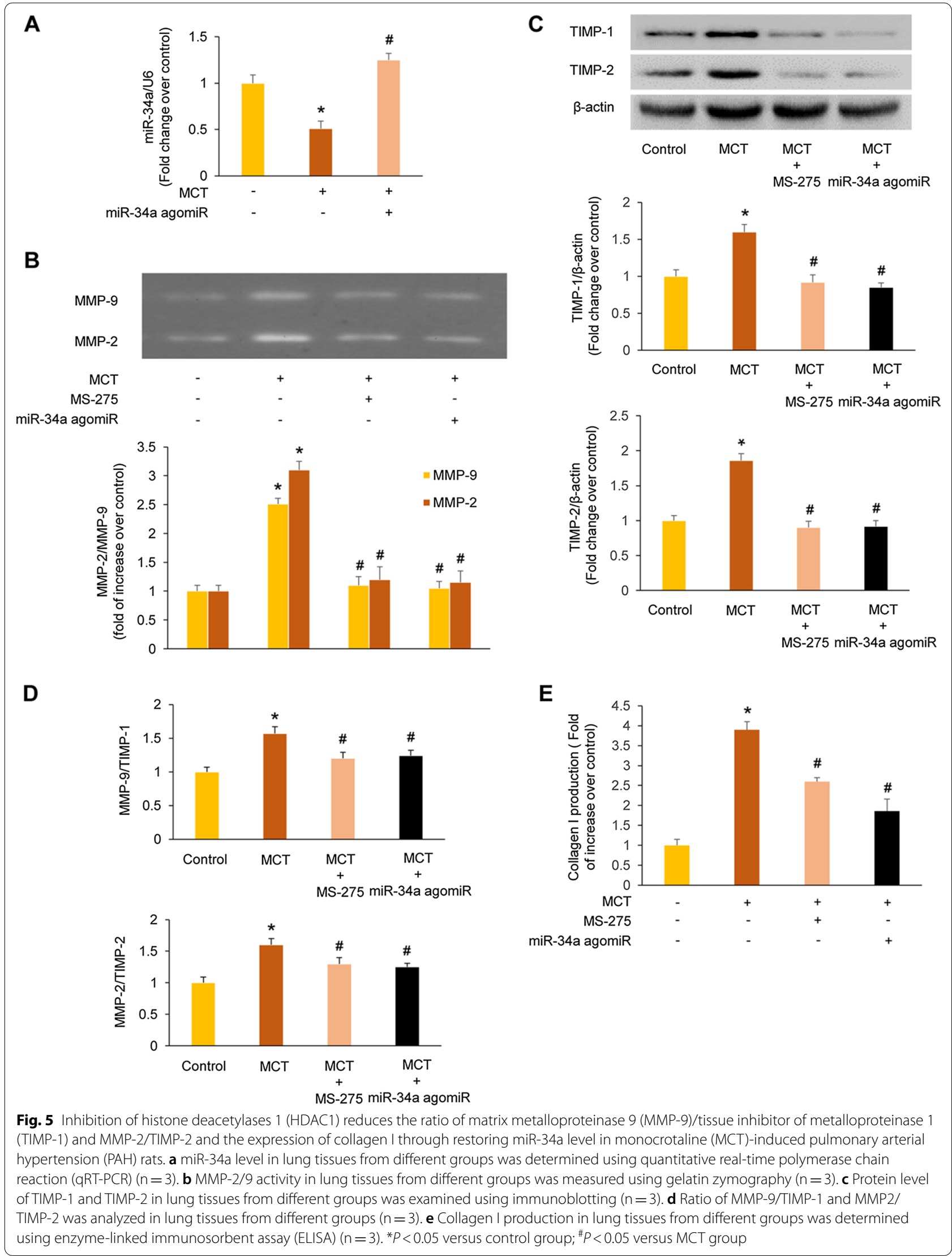


lung vascular remodeling induced by MCT with a reduction of wall thickness in arteries, as presented in Fig. 6A. Quantitative morphometric analysis showed that the percentage of medial wall thickness (\%MT) for lung vascular with diameters greater than $50 \mu \mathrm{m}$ was increased from $11.34 \pm 3.25 \%$ in control rats to $67.62 \pm 6.60 \%$ in MCTtreated rats $(P<0.05)$, while administration of MS-275 and miR-34a agomiR reduced the \%MT to $32.10 \pm 4.95 \%$ and $20.43 \pm 5.02 \%$, respectively (both $P<0.05$ versus MCT group). In addition, for lung vascular with diameters less than $50 \mu \mathrm{m}$, the \%MT was elevated from $15.55 \pm 4.52 \%$ in control rats to $75.26 \pm 5.10 \%$ in MCTtreated rats $(P<0.05)$, while administration of MS-275 and miR-34a agomiR reduced the \%MT to $35.68 \pm 4.35 \%$ and $21.89 \pm 4.62 \%$, respectively (both $P<0.05$ versus MCT group), as shown in Fig. 6B. Meanwhile, collagen deposition was determined by Masson's staining to evaluate the accumulation of ECM components in pulmonary arteries. As indicated in Fig. 6C, collagen deposition in pulmonary vessels with diameters no matter greater than $50 \mu \mathrm{m}$ or less than $50 \mu \mathrm{m}$ was significantly increased in the lung tissues of MCT-treated rats compared with control rats. However, the presence of MS-275 or miR-34a agomiR reduced the collagen deposition in pulmonary vessels in MCT-treated rats dramatically. In addition, as depicted in Fig. 6D, treatment with miR-34a agomiR after MCT injection dramatically reduced RVSP from $56.45 \pm 2.54 \mathrm{mmHg}$ in MCT-treated

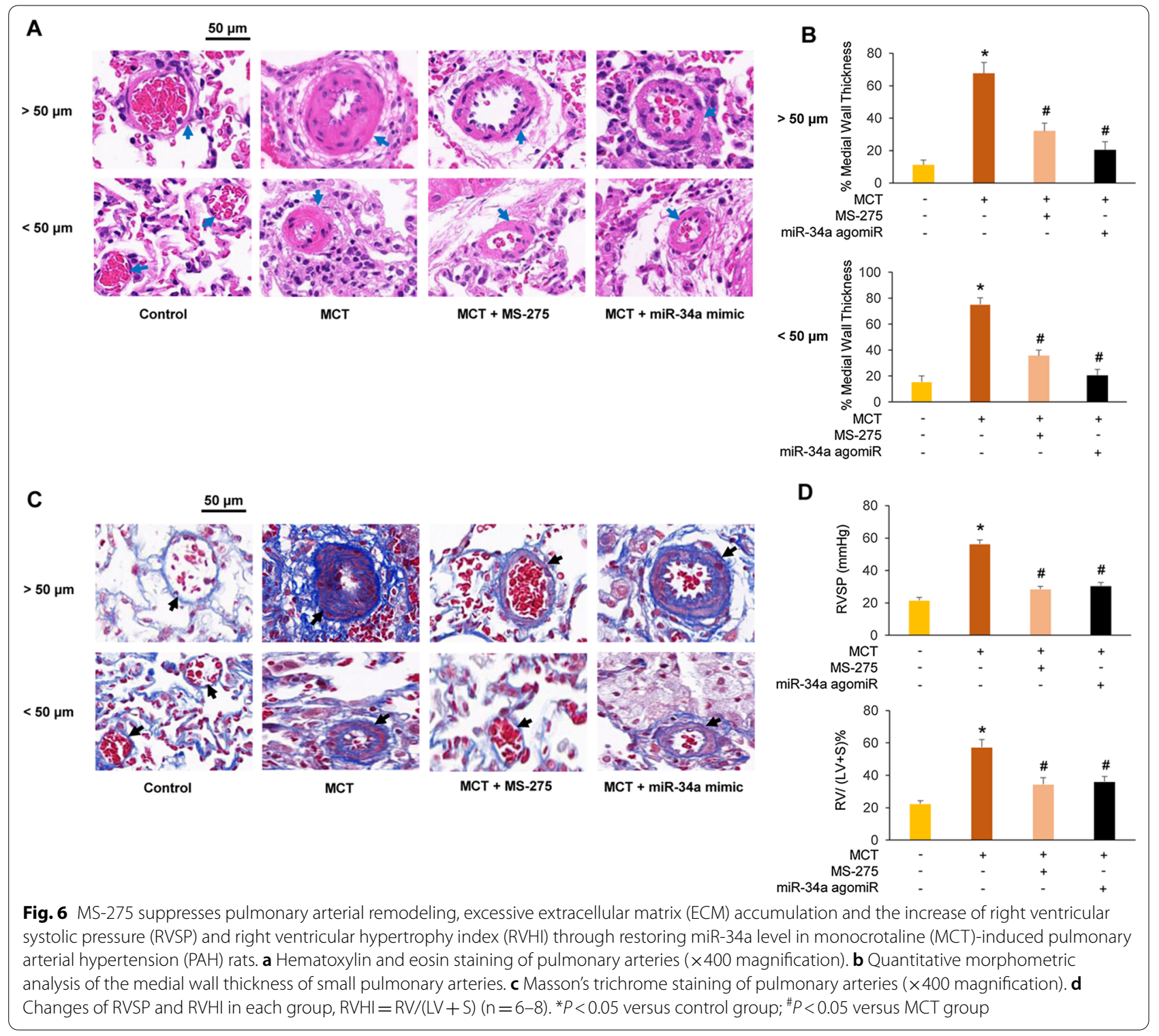


rats to $30.56 \pm 2.14 \mathrm{mmHg}(P<0.05$ versus MCT group) and reversed the $\mathrm{RV} /(\mathrm{LV}+\mathrm{S})$ ratio from $0.57 \pm 0.05$ in MCT-induced PAH rats to $0.36 \pm 0.03(P<0.05$ versus MCT group). These results suggest that inhibition of HDAC1 alleviates pulmonary arterial remodeling, excessive ECM accumulation and the increase of RVSP and RVHI through restoring miR-34a level in MCT-induced PAH rats.

\section{Discussion}

In the present study, we have demonstrated that HDAC1 expression is up-regulated in the lung tissues of MCTinduced PAH rat models, which is coupled to reduction of miR-34a level and elevation of MMP-9/TIMP-1 and MMP-2/TIMP-2 and subsequent increase of collagen I protein. In addition, inhibition of HDAC 1 by MS-275 and over-expression of miR-34a by miR-34a agomiR effectively ameliorate excessive collagen I production and pulmonary arterial remodeling, preventing the development of MCT-induced PAH, as shown in Fig. 7. Our results suggest that inhibition of HDAC1 modulates of excessive ECM accumulation and pulmonary vascular remodeling through up-regulation of miR-34a, being of potential value in the management of $\mathrm{PAH}$.

HDAC1 is recruited by multiple transcriptional regulators to a specific genomic region to mediate the repression of corresponding target genes via deacetylation of substrates [21]. Studies have found that HDAC1 plays a vital role in cell proliferation and differentiation, cell cycle regulation as well as tissue transformation and development [22-26]. In addition, HDAC1 is found increased in various cancers, nervous system and heart diseases, and so on [27-29]. HDAC1 level is also elevated in lungs from human idiopathic $\mathrm{PAH}$ and rats with PAH; class I HDACs participate in the ECM remodeling of various tissues through different molecular mechanisms $[8,9,30]$. In this study, we used a selective class I HDAC inhibitor MS-275 to examine the effect of HDAC1 on ECM accumulation in pulmonary arteries. Our results demonstrate that inhibition of HDAC1 ameliorates MCT-induced rat excessive ECM accumulation and pulmonary vascular remodeling, indicating that HDAC1 might play an indispensable role in PAH development.

miR-34a causes degradation or translational repression of the target gene through binding to the $3^{\prime}$ untranslated region of mRNAs [31]. miR-34a has been found to participate in various important biological processes, such as cell apoptosis, differentiation and proliferation, as well as tumor suppression, development and metabolism [32-35]. Recent studies have also found that miR-34a level is decreased in the lung tissue of patients and various animal models with $\mathrm{PAH}$; overexpression of miR-34a reverses hypoxia or MCTinduced pulmonary vascular remodeling and $\mathrm{PAH}[17$, 18], suggesting the important role of miR-34a in PAH development. Studies have further shown that HDAC1 inhibits the expression of miR-34a in two ways: HDAC1 binds to the promoter region of miR-34a to directly inhibit gene transcription through deacetylation [15]; HDAC1 directly acts on p53, one upstream gene of miR-34a, and subsequently suppresses miR-34a level [16]. Our present study indicated that both inhibition of HDAC1 activity with MS-275 in MCT-induced PAH rat and loss of HDAC1 with siRNA in PASMCs up-regulated expression level of miR-34a. These results suggest

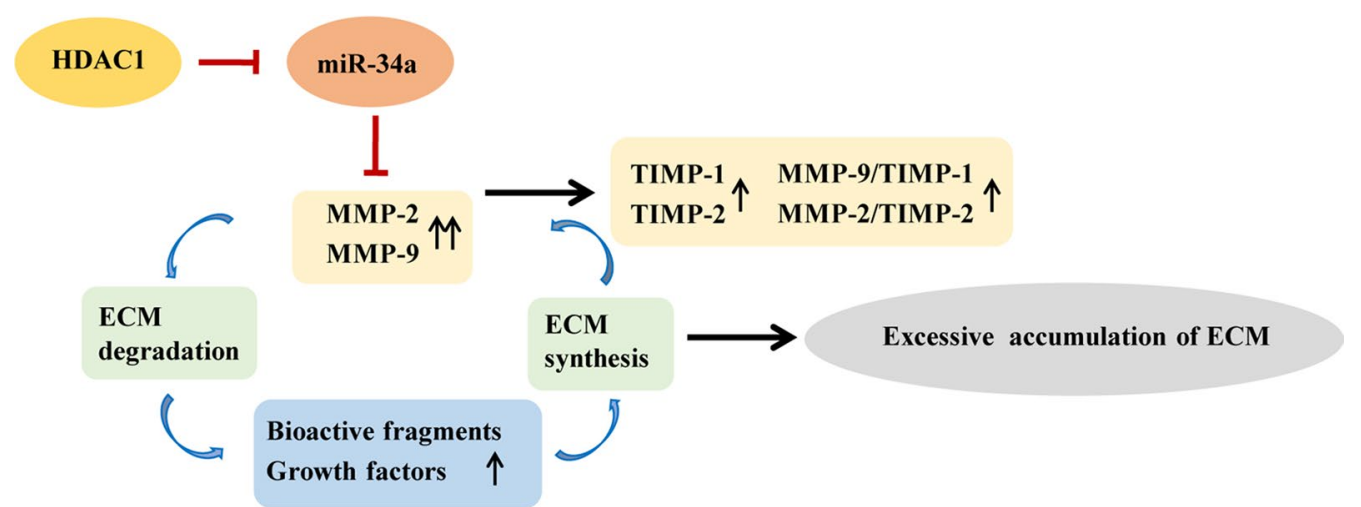

Fig. 7 Proposed mechanism for inhibition of histone deacetylases 1 (HDAC1) alleviating monocrotaline (MCT)-induced pulmonary arterial hypertension (PAH). Injection of MCT up-regulates HDAC1 expression, reduces miR-34a level, and subsequently increases the activity of matrix metalloproteinase 2/9 (MMP-2/9). The elevated activity of MMP-2/9 initiates degradation of extracellular matrix (ECM) and finally leads to excessive ECM accumulation through a vicious circle. In addition, tissue inhibitor of metalloproteinase 1/2 (TIMP-1/2) expression is also up-regulated to inhibit excessive MMP activity through a balancing mechanism. However, since the balancing effect is limited, the ratio of MMP-9/TIMP-1 and MMP-2/ TIMP-2 is elevated. Inhibition of HDAC1 reverses the process in MCT-induced PAH through restoring miR-34a level 
that miR-34a might be a direct target of HDAC1 in pulmonary vascular remodeling, which needs further demonstration in our future studies.

MMP-2 and MMP-9 belong to ECM proteolytic enzymes, which are responsible for collagen degradation, particularly for collagen I degradation [36]. TIMP-1 and TIMP-2 are the small molecular glycoproteins which inhibit the activity of MMP-2 and MMP-9 [37]. Studies have found that elevated MMP activity and consequent ECM remodeling in pulmonary vasculature are involved in the progression of PAH in both experimental models and patients [38-40]. The decreased expression of miR-34a promotes the increased activity of MMP-2 and MMP-9 [41, 42], which is consistent with our present study. Injection of MCT causes response of inflammatory cells and injury of endothelial cells in lungs, which secrete and activate more MMP-2 and MMP-9. MMP-2 and MMP-9 initiate degradation of ECM and subsequent release of bioactive ECM fragments and growth factors, which in turn synthesize and secrete more ECM components. The dysregulated ECM metabolism finally leads to abnormal ECM remodeling through a vicious circle. During this time, the expression of TIMP-1 and TIMP-2 are also up-regulated to inhibit excessive MMP activity through a balancing mechanism [39]. However, since the balancing effect is limited, the ratio of MMP-9/TIMP-1 and MMP-2/ TIMP-2 is elevated, which contributes to excessive ECM accumulation and the development of PAH [43].

\section{Conclusion}

The present study demonstrated that inhibition of HDAC1 effectively ameliorates excessive ECM accumulation and pulmonary vascular remodeling in MCTinduced rat $\mathrm{PAH}$, suggesting that inhibiting HDAC1 might be a novel therapeutic strategy in the prevention and treatment of PAH. However, the safety and effectiveness of HDAC1 inhibitors still need to be tested and verified in the further studies.

\begin{abstract}
Abbreviations
\%MT: Percentage of medial wall thickness; ECM: Extracellular matrix; ELISA: Enzyme-linked immunosorbent assay; HATs: Histone acetyltransferases; HDAC: Histone deacetylases; HE: Hematoxylin and eosin; LV + S: Left ventricle plus interventricular septum; MCT: Monocrotaline; MMP-2/9: Matrix metalloproteinase 2/9; PAH: Pulmonary arterial hypertension; PASMCs: Pulmonary arterial smooth muscle cells; qRT-PCR: Quantitative real-time polymerase chain reaction; RV: Right ventricle; RVHI: Right ventricle hypertrophy index; RVSP: Right ventricular systolic pressure; TIMP-1/2: Tissue inhibitor of metalloproteinase $1 / 2$.
\end{abstract}

\section{Acknowledgements}

We thank professor Manxiang Li for his guidance on the writing of this paper.

\section{Authors' contributions}

FWL and YXW participated in research design. XLS, DW and LC conducted experiments. DW, HW and LJC contributed new reagents or analytic tools. FWL and HW carried out the statistical analysis. FWL and HW performed data analysis. FWL and YXW wrote or contributed to the writing of the manuscript. All authors read and approved the final manuscript.

\section{Funding}

This work was supported by the Natural Science Foundation of China (No. 81960014); and Cuiying Scientific and Technological Innovation Program of Lanzhou University Second Hospital (No. CY2018-QN12).

\section{Availability of data and materials}

All data generated or analyzed during this study are included in this article.

\section{Declarations}

Ethics approval and consent to participate

All animal experiments were approved by Laboratory Animal Care Committee of Lanzhou University Second Hospital (Permission Number: D2019-030) and performed in accordance with the Guidelines for Animal Experimentation of Lanzhou University Second Hospital.

\section{Consent for publication}

Not applicable.

\section{Competing interests}

The authors declare that they have no competing interests.

Received: 18 April 2021 Accepted: 24 August 2021

Published online: 31 August 2021

\section{References}

1. Simonneau G, Montani D, Celermajer DS, Denton CP, Gatzoulis MA, Krowka M, et al. Haemodynamic definitions and updated clinical classification of pulmonary hypertension. Eur Respir J. 2019;53(1):1801913.

2. Guignabert C, Dorfmüller P. Pathology and pathobiology of pulmonary hypertension. Semin Respir Crit Care Med. 2017;38(5):571-84.

3. Thenappan T, Chan SY, Weir EK. Role of extracellular matrix in the pathogenesis of pulmonary arterial hypertension. Am J Physiol Heart Circ Physiol. 2018;315(5):H1322-31.

4. Frangogiannis NG. Fibroblasts and the extracellular matrix in right ventricular disease. Cardiovasc Res. 2017;113(12):1453-64.

5. Li G, Tian Y, Zhu WG. The roles of histone deacetylases and their inhibitors in cancer therapy. Front Cell Dev Biol. 2020;8:576946.

6. Verza FA, Das U, Fachin AL, Dimmock JR, Marins M. Roles of histone deacetylases and inhibitors in anticancer therapy. Cancers (Basel). 2020;12(6):1664.

7. Wang P, Wang Z, Liu J. Role of HDACs in normal and malignant hematopoiesis. Mol Cancer. 2020;19(1):5.

8. Bombardo M, Chen R, Malagola E, Saponara E, Hills AP, Graf R, et al. Inhibition of class i histone deacetylases abrogates tumor growth factor $\beta$ expression and development of fibrosis during chronic pancreatitis. Mol Pharmacol. 2018;94(2):793-801.

9. Kim DJ, Dunleavey JM, Xiao L, Ollila DW, Troester MA, Otey CA, et al. Suppression of TGF $\beta$-mediated conversion of endothelial cells and fibroblasts into cancer associated (myo)fibroblasts via hdac inhibition. Br J Cancer. 2018;118(10):1359-68.

10. Kawabata T, Nishida K, Takasugi K, Ogawa H, Sada K, Kadota Y, et al. Increased activity and expression of histone deacetylase 1 in relation to tumor necrosis factor-alpha in synovial tissue of rheumatoid arthritis. Arthritis Res Ther. 2010;12(4):R133.

11. Chelladurai P, Dabral S, Basineni SR, Chen CN, Schmoranzer M, Bender $\mathrm{N}$, et al. Isoform-specific characterization of class i histone deacetylases and their therapeutic modulation in pulmonary hypertension. Sci Rep. 2020;10(1):12864. 
12. Zhao L, Chen CN, Hajji N, Oliver E, Cotroneo E, Wharton J, et al. Histone deacetylation inhibition in pulmonary hypertension: therapeutic potential of valproic acid and suberoylanilide hydroxamic acid. Circulation. 2012;126(4):455-67.

13. Wang J, Saren G, Jiang H. Hdac inhibition: a novel therapeutic target for attenuating pulmonary hypertension by regulating tregs. Int J Cardiol. 2015;198:176-7

14. Mani SK, Kern CB, Kimbrough D, Addy B, Kasiganesan H, Rivers WT, et al. Inhibition of class i histone deacetylase activity represses matrix metalloproteinase-2 and -9 expression and preserves Iv function postmyocardial infarction. Am J Physiol Heart Circ Physiol. 2015;308(11):H1391-401.

15. Wang $L, B u P, A i Y$, Srinivasan T, Chen HJ, Xiang K, et al. A long non-coding RNA targets microRNA miR-34a to regulate colon cancer stem cell asymmetric division. Elife. 2016;5:e14620.

16. Lin L, Jiang H, Huang M, Hou X, Sun X, Jiang X, et al. Depletion of histone deacetylase 1 inhibits metastatic abilities of gastric cancer cells by regulating the miR-34a/CD44 pathway. Oncol Rep. 2015;34(2):663-72.

17. Chen KH, Dasgupta A, Lin J, Potus F, Bonnet S, Iremonger J, et al. Epigenetic dysregulation of the dynamin-related protein 1 binding partners MID49 and MID51 increases mitotic mitochondrial fission and promotes pulmonary arterial hypertension: mechanistic and therapeutic implications. Circulation. 2018;138(3):287-304.

18. Wang $P, X u J$, Hou Z, Wang F, Song Y, Wang J, et al. miRNA-34a promotes proliferation of human pulmonary artery smooth muscle cells by targeting PDGFRA. Cell Prolif. 2016;49(4):484-93.

19. Fan YF, Zhang R, Jiang X, Wen L, Wu DC, Liu D, et al. The phosphodiesterase-5 inhibitor vardenafil reduces oxidative stress while reversing pulmonary arterial hypertension. Cardiovasc Res. 2013;99(3):395-403.

20. Rathinasabapathy A, Bruce E, Espejo A, Horowitz A, Sudhan DR, Nair A et al. Therapeutic potential of adipose stem cell-derived conditioned medium against pulmonary hypertension and lung fibrosis. Br J Pharmacol. 2016;173(19):2859-79.

21. Reichert N, Choukrallah MA, Matthias P. Multiple roles of class I HDACs in proliferation, differentiation, and development. Cell Mol Life Sci. 2012;69(13):2173-87.

22. Huang $J H, X u Y$, Lin FY. The inhibition of microRNA-326 by SP1/HDAC1 contributes to proliferation and metastasis of osteosarcoma through promoting SMO expression. J Cell Mol Med. 2020;24(18):10876-88.

23. Preglej $T$, Hamminger $P$, Luu M, Bulat T, Andersen L, Göschl L, et al. Histone deacetylases 1 and 2 restrain CD4+ cytotoxic T lymphocyte differentiation. JCl Insight. 2020;5(4):e133393.

24. Liu H, Chen S, Yao X, Li Y, Chen CH, Liu J, et al. Histone deacetylases 1 and 2 regulate the transcriptional programs of nephron progenitors and renal vesicles. Development. 2018;145(10):dev153619.

25. Thambyrajah R, Fadlullah MZH, Proffitt M, Patel R, Cowley SM, Kouskoff V, et al. HDAC1 and HDAC2 modulate TGF- $\beta$ signaling during endothelialto-hematopoietic transition. Stem Cell Rep. 2018;10(4):1369-83.

26. Tang $T$, Zhang Y, Wang Y, Cai Z, Lu Z, Li L, et al. HDAC1 and HDAC2 regulate intermediate progenitor positioning to safeguard neocortical development. Neuron. 2019;101(6):1117-33 e5.

27. Witt AE, Lee CW, Lee TI, Azzam DJ, Wang B, Caslini C, et al. Identification of a cancer stem cell-specific function for the histone deacetylases, HDAC 1 and HDAC7, in breast and ovarian cancer. Oncogene. 2017;36(12):1707-20.

28. Datta M, Staszewski O, Raschi E, Frosch M, Hagemeyer N, Tay TL, et al. Histone deacetylases 1 and 2 regulate microglia function during development, homeostasis, and neurodegeneration in a contextdependent manner. Immunity. 2018;48(3):514-29 e6.

29. Jia S, Yang S, Du P, Gao K, Cao Y, Yao B, et al. Regulatory factor X1 downregulation contributes to monocyte chemoattractant protein-1 overexpression in CD14+ monocytes via epigenetic mechanisms in coronary heart disease. Front Genet. 2019;10:1098.

30. Lan B, Hayama E, Kawaguchi N, Furutani Y, Nakanishi T. Therapeutic efficacy of valproic acid in a combined monocrotaline and chronic hypoxia rat model of severe pulmonary hypertension. PLOS ONE. 2015;10(1):e0117211.

31. Slabáková E, Culig Z, Remšík J, Souček K. Alternative mechanisms of miR34a regulation in cancer. Cell Death Dis. 2017;8(10):e3100.

32. Iwagami Y, Zou J, Zhang H, Cao K, Ji C, Kim M, et al. Alcohol-mediated miR-34a modulates hepatocyte growth and apoptosis. J Cell Mol Med. 2018;22(8):3987-95.

33. Wang B, Li D, Kovalchuk I, Apel IJ, Chinnaiyan AM, Wóycicki RK, et al. miR-34a directly targets tRNA(i)(Met) precursors and affects cellular proliferation, cell cycle, and apoptosis. Proc Natl Acad Sci USA. 2018;115(28):7392-7.

34. Jiang L, Hermeking H. miR-34a and miR-34b/c suppress intestinal tumorigenesis. Cancer Res. 2017;77(10):2746-58.

35. Dong $\mathrm{P}$, Xiong Y, Watari H, Hanley SJ, Konno Y, Ihira K, et al. miR-137 and miR-34a directly target snail and inhibit EMT, invasion and sphere-forming ability of ovarian cancer cells. J Exp Clin Cancer Res. 2016;35(1):132.

36. Cheng Z, Limbu MH, Wang Z, Liu J, Liu L, Zhang X, et al. MMP-2 and 9 in chronic kidney disease. Int J Mol Sci. 2017;18(4):776.

37. Raeeszadeh-Sarmazdeh M, Greene KA, Sankaran B, Downey GP, Radisky DC, Radisky ES. Directed evolution of the metalloproteinase inhibitor TIMP-1 reveals that its $\mathrm{N}$ - and C-terminal domains cooperate in matrix metalloproteinase recognition. J Biol Chem. 2019;294(24):9476-88.

38. Zelko IN, Zhu J, Ritzenthaler JD, Roman J. Pulmonary hypertension and vascular remodeling in mice exposed to crystalline silica. Respir Res. 2016;17(1):160.

39. Chelladurai P, Seeger W, Pullamsetti SS. Matrix metalloproteinases and their inhibitors in pulmonary hypertension. Eur Respir J. 2012:40(3):766-82.

40. Safdar Z, Tamez E, Chan W, Arya B, Ge Y, Deswal A, et al. Circulating collagen biomarkers as indicators of disease severity in pulmonary arterial hypertension. JACC Heart Fail. 2014;2(4):412-21.

41. Li YY, Tao YW, Gao S, Li P, Zheng JM, Zhang SE, et al. Cancer-associated fibroblasts contribute to oral cancer cells proliferation and metastasis via exosome-mediated paracrine miR-34a-5p. EBioMedicine. 2018;36:209-20.

42. Córdova-Rivas S, Fraire-Soto I, Mercado-Casas Torres A, Servín-González LS, Granados-López AJ, López-HernándezY, et al. $5 p$ and 3p strands of miR-34 family members have differential effects in cell proliferation, migration, and invasion in cervical cancer cells. Int J Mol Sci. 2019;20(3):545

43. Safdar Z, Frost A, Basant A, Deswal A, O'Brian Smith E, Entman M. Spironolactone in pulmonary arterial hypertension: results of a cross-over study. Pulm Circ. 2020;10(2):2045894019898030.

\section{Publisher's Note}

Springer Nature remains neutral with regard to jurisdictional claims in published maps and institutional affiliations. 